\title{
Human Dental Pulp Stem Cells via the NF-kB Pathway
}

\author{
Shensheng Gu Shujun Ran Feng Qin Dong Cao Jia Wang Bin Liu \\ Jingping Liang
}

Department of Endodontics and Operative Dentistry, Ninth People's Hospital, School of Medicine, Shanghai Jiaotong University, Shanghai, China and Shanghai Key Laboratory of Stomatology, Shanghai Research Institute of Stomatology, Ninth People's Hospital, School of Medicine, Shanghai Jiaotong University, Shanghai, China

\section{Key Words}

ZBTB20 • HDPSCs • Odontogenic differentiation • Nuclear factor-kappaB

\begin{abstract}
Background/Aims: Odontogenic differentiation of human dental pulp stem cells (HDPSCs) is regulated by multiple factors and signaling molecules. However, their regulatory mechanisms are not completely understood. In this study, we investigated the role of Zinc finger and BTB domain-containing 20 (ZBTB20) in odontoblastic differentiation of HDPSCs. Methods: HDPSCs were obtained from human third molars and ZBTB20 expression was examined by qRT-PCR and western blot. Their osteo/odontogenic differentiation and the involvement of NF-KB pathway were subsequently investigated. Results: The expression of ZBTB20 is upregulated in a time-dependent manner during odontogenic differentiation of hDPSCs. Inhibition of ZBTB20 reduced osteogenic medium (OM)-induced odontogenic differentiation, reflected in decreased alkaline phosphatase (ALP) activity, mineralized nodule formation and mRNA expression of odonto/osteogenic marker genes. In contrast, overexpression of ZBTB20 enhanced ALP activity, mineralization and the expression of differentiation marker genes. Furthermore, the expression of IKB $\alpha$ was increased by ZBTB20 silencing in HDPSCs, whereas ZBTB20 overexpression decreased IKB $\alpha$ and enhanced nuclear NF-KB p65. Inhibition of the NF- $k B$ pathway significantly suppressed the odontogenic differentiation of HDPSCs induced by ZBTB20. Conclusion: This study shows for the first time that ZBTB20 plays an important role during odontoblastic differentiation of HDPSCs and may have clinical implications for regenerative endodontics.
\end{abstract}




\section{Cellular Physiology Cell Physiol Biochem 2015;36:1725-1734 \begin{tabular}{ll|l} 
and $10.1159 / 000430145$ & $\begin{array}{l}\text { D 2015 S. Karger AG, Basel } \\
\text { www.karger.com/cpb }\end{array}$ \\
\hline
\end{tabular} \\ Gu et al.: ZBTB20 Regulates Odontogenic Differentiation of hDPSCs}

\section{Introduction}

Human dental pulp stem cells (HDPSCs) are multipotent cells that were originally isolated from adult human dental pulp tissue [1]. These cells are capable of differentiating into multiple cell lineages, including odontoblasts [2], osteoblasts [3], adipocytes [4], chondrocytes [4], myocytes [5] and neuronal cells [4]. DPSCs are thought to possess great therapeutic potential for repairing damaged and/or defective dentinogenesis [6]. However, the lack of detailed understanding of odontoblast/osteoblast-like differentiation of HDPCs limits their application in dental tissue engineering. Consequently, a better understanding of the molecular mechanisms regulating differentiation of HDPCs would be of great significance.

Zinc finger and BTB domain-containing 20 (ZBTB20), also termed DPZF, HOF or ZNF288 [7], belongs to a subfamily of zinc finger proteins containing C2H2 Krüppel-type zinc fingers and BTB/POZ domains. It belongs to a class of nuclear DNA-binding transcription factors which are implicated in many developmental processes [8]. ZBTB20 knockout mice display abnormal glucose homeostasis, impaired hormonal responses, and depletion of energy stores, consistent with an energetic deficit [9]. ZBTB20 knockdown in a pancreatic $\beta$ cell line impairs glucose-stimulated insulin secretion [10]. Furthermore, ZBTB20 regulates nociception and pain sensation in nociceptive sensory neurons [11]. Zhang et al. found that ZBTB20 shares significant homology to ZBTB16, with an overall identity of 30\% [12]. Previous studies have demonstrated that ZBTB16 is involved in osteoblastic differentiation of human mesenchymal stem cells (hMSCs) [13]. For example, Ikeda et al. showed that ZBTB16 acts an upstream regulator of RUNX2 in osteoblastic differentiation of hMSCs [14]. Silencing of ZBTB16 reduced the expression of osteoblast-specific genes such as alkaline phosphatase (ALP), collagen 1A1, RUNX2/CBFA1, and osteocalcin (OCN) in the presence of osteogenic differentiation medium in hMSCs [15]. A recent study demonstrated that, in dental follicle cells, ZBTB16 induces the expression of osteogenic differentiation marker genes [16]. Thus, we hypothesized that ZBTB20 might play similar important roles in odontogenic/ osteoblastic differentiation of HDPSCs. Choi et al. found that expression of ZBTB20 increases during odontoblast/osteoblast differentiation of HDPSCs [17]. However, the role of ZBTB20 and its mechanism of action in osteoblastic differentiation remain unclear.

In the present study, we investigated the role of ZBTB20 in odontoblastic differentiation of HDPSCs. Our findings suggest that exogenous ZBTB20 application may induce the odontogenic/osteoblastic differentiation of HDPSCs via activation of the NF-кB signaling pathway.

\section{Materials and Methods}

\section{Cell culture}

Normal human third molars extracted from adults (18-26 years of age) at Ninth People's Hospital were used as the source of stem cells. All of the experimental protocols involving human tissues were approved by the Ethics Committee of Ninth People's Hospital, School of Medicine, Shanghai Jiao Tong University. Dental pulp stem cells were isolated as described previously [1]. The obtained cells were cultured in alpha-minimum essential medium ( $\alpha$-MEM; Hyclone, Logan, UT, USA) supplemented with 10\% FBS, $100 \mathrm{U} / \mathrm{mL}$ penicillin, and $100 \mu \mathrm{g} / \mathrm{mL}$ streptomycin in a humidified atmosphere of $5 \% \mathrm{CO}_{2}$. The medium was replaced every 2 days. Cells were subcultured at the ratio of 1:3 until they reached $75-85 \%$ confluence. HDPSCs below passage 5 were used in this study. For odontogenic differentiation, osteogenic medium (OM, $\alpha$-MEM, 10\% FBS, 10 mM $\beta$-glycerophosphate, $50 \mathrm{mM}$ ascorbic acid, and $0.1 \mathrm{mM}$ dexamethasone) was used to induce differentiation.

RNA extraction and quantitative real-time RT-PCR

Total RNA was isolated using Trizol reagent (Invitrogen, Carlsbad, CA, USA) according to the manufacturer's protocol. cDNA was synthesized using the PrimeScript RT reagent Kit (TaKaRa, Dalian, China). Human alkaline phosphatase (ALP), osteocalcin (OCN), dentin sialophosphoprotein (DSPP), collagen I (Col I) runt-related transcription factor 2 (RUNX2) and ZBTB20 transcripts were quantified by qRT-PCR 


\section{Cellular Physiology Cell Physiol Biochem 2015;36:1725-1734

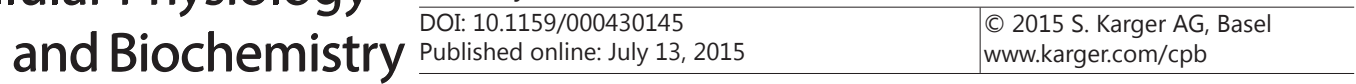 \\ Gu et al.: ZBTB20 Regulates Odontogenic Differentiation of hDPSCs}

using the SYBR Premix Ex Taq II kit (TaKaRa) and the Applied Biosystems ABI Prism 7500 HT sequence detection system (Applied Biosystems, Foster City, CA, USA). GAPDH mRNA was amplified as an internal control. Expression of mRNA was evaluated by the $2^{-\triangle \Delta C T}$ method. All reactions were run in triplicate.

\section{Measurement of ALP activity and Alizarin red S staining}

The activity of ALP was determined with an ALP activity kit (Sigma-Aldrich, St Louis, MO, USA), according to the manufacturer's protocol. Signals were normalized based on protein concentration [18]. The mineralization of HDPSCs was evaluated by Alizarin Red S staining. At 14 days after OM stimulation, the cells were fixed with 70\% ethanol for 10 min, stained with an Alizarin Red Solution (2\%, pH 4.2) for 10 min and washed with distilled water to remove the unbound stain. Cultures stained with Alizarin Red $\mathrm{S}$ were photographed with a digital camera. Then, cells stained with Alizarin Red were destained with 10 $\%$ cetylpyridinium chloride in $10 \mathrm{Mm}$ sodium phosphate for $30 \mathrm{~min}$. The mineralization was quantified by measuring absorbance at $562 \mathrm{~nm}$. All experiments were performed in triplicate.

\section{Lentivirus production and transduction}

The coding sequences of ZBTB20 and its shRNA were purchased from Hanbio (Shanghai, China), and inserted into pWPI-GFP and pLL3.7 vectors, respectively. Lentivirus production and infection were performed as previously described [22]. HDPSCs were infected with recombinant lentivirus-transducing units in the presence of $8 \mu \mathrm{g} / \mathrm{mL}$ polybrene. Cells were assayed $48 \mathrm{~h}$ after transfection. Thereafter, the medium was changed to OM. ALP activity was measured 3 and 7 days later, and cultures were stained with Alizarin Red S after 2 weeks.

\section{Immunofluorescence assay}

The effect of ZBTB20 on the nuclear translocation of the NF-KB p65 subunit was examined by immunofluorescence assay. At 14 days after OM stimulation, cells were fixed in $4 \%$ paraformaldehyde in PBS for $30 \mathrm{~min}$ at room temperature. After washing in PBS, cells were incubated in $0.25 \%$ TritonX-100 for permeabilization and treated with $10 \%$ normal goat serum for $1 \mathrm{~h}$ at room temperature. Afterwards, the cells were sequentially incubated with anti-p65 antibody (Cell Signaling Technology, Danvers, MA, USA) followed by fluorescein isothiocyanate (FITC)-conjugated IgG. Nuclei were stained with DAPI for $3 \mathrm{~min}$ at room temperature. After washing with PBS, staining was observed using a confocal laser microscope (Carl Zeiss, Oberkochen, Germany) and photographed.

\section{Western blot analysis}

Cells were washed with PBS and lysed with lysis buffer (Beyotime, Nanjing, China). To measure the expressions of NF-kB pathway proteins, total protein and nucleoprotein were collected separately as previously described [19]. The protein concentrations of samples were determined using the BCA assay (Pierce, Rockford, IL, USA). Separation of protein bands was performed by $10 \%$ SDS-PAGE and proteins were electrically transferred onto a PVDF membrane (Roche, Basel, Switzerland). Membranes were incubated with primary antibodies against ZBTB20 (Sigma), p65 and IкB $\alpha$ (Santa Cruz Biotechnology, Santa Cruz, CA, USA) overnight at $4^{\circ} \mathrm{C}$. Lamin-B and $\beta$-actin (both from Santa Cruz Biotechnology) were used as markers. After the membranes were incubated with HRP-conjugated secondary antibody for $1 \mathrm{~h}$, protein bands were visualized using an ECL chemiluminescence detection system. Densitometry was analyzed using the ImageJ software program (NIH, Bethesda, MD, USA).

\section{Statistical Analysis}

Data are expressed as mean \pm SD from three independent experiments and differences among groups were assessed using Student's two-tailed $t$ test. $P<0.05$ was considered significant.

\section{Results}

ZBTB20 is upregulated during odontogenic differentiation of HDPSCs

To investigate the expression of ZBTB20 mRNA and protein during odontogenic differentiation of HDPSCs, cells were cultured in OM for 2 weeks, after which samples were 


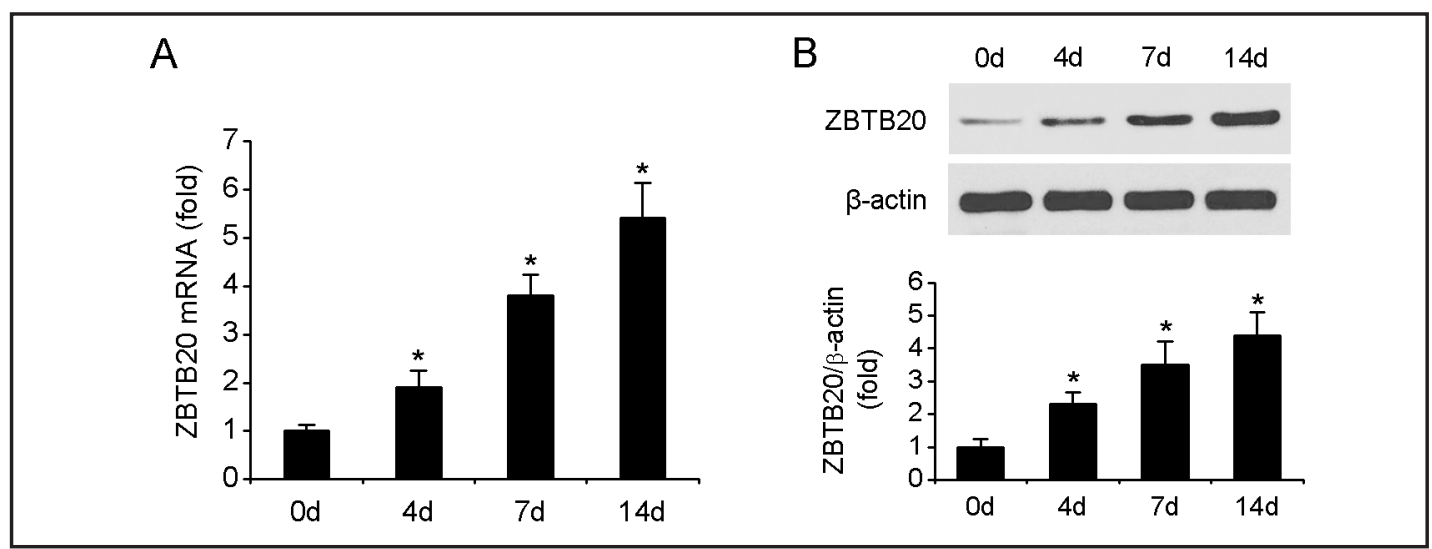

Fig. 1. ZBTB20 is upregulated during odontogenic differentiation of HDPSCs. Cells were cultured in OM for $0,4,7$ or 14 days. (A) ZBTB20 mRNA expression was analyzed by qRT-PCR. $\beta$-actin was used as an internal control. (B) ZBTB20 protein expression was analyzed by western blot. Protein levels were normalized as a ratio to $\beta$-actin after densitometric quantification and presented as fold change relative to the control group. Values are presented as mean \pm SD for three independent experiments. ${ }^{*} P<0.05 v s .0$ day group.

analyzed by qRT-PCR and western blot at day $0,4,7$, and 14 after induction of differentiation. Results showed that expression of ZBTB20 mRNA and protein was significantly increased throughout this period (Fig. 1A and B), suggesting that ZBTB20 may be important for differentiation of HDPSCs into odontoblasts.

Inhibition of ZBTB20 impairs odontogenic differentiation of HDPSCs

To explore the role of ZBTB20 in odontogenic differentiation of HDPSCs, HDPSCs were transfected with lentiviral constructs containing ZBTB20 shRNA or negative control, and the expression of ZBTB20 was analyzed by western blot (Fig. 2A). These cells were then cultured in OM to induce odontogenic differentiation. We found that ALP activity, one of the earliest markers of odontoblast differentiation, was significantly reduced at 3 and 7 days in HDPSCs with silenced ZBTB20 compared to control cells (Fig. 2B). Consistently, qRT-PCR results showed that the odonto/osteogenic marker genes ALP, OCN, DSPP and Col I were strongly inhibited in ZBTB20-depleted cells at 7 days after induction (Fig. 2C). The key transcription factor RUNX2, which regulates odonto/osteogenic differentiation, was also significantly downregulated at 7 and 14 days after induction in ZBTB20-depleted cells compared to control cells (Fig. 2E). Furthermore, Alizarin red staining revealed that mineralization was significantly lower in cells with ZBTB20 suppression at 14 days (Fig. 2D). These data suggest that ZBTB20 is required for the odontogenic differentiation of HDPSCs.

Overexpression of ZBTB20 promotes odontogenic differentiation of HDPSCs

We further examined the effect of ZBTB20 overexpression on odontoblastic differentiation of HDPSCs. ZBTB20 overexpressing lentiviruses were transduced into HDPSCs, and overexpression of ZBTB20 was confirmed by western blot (Fig. 3A). The results showed that introduction of ZBTB20 markedly increased ALP activity, odonto/osteogenic marker gene expression and matrix mineralization in ZBTB20-overexpressing HDPSCs compared with negative controls during the odontogenic differentiation process (Fig. 3B-D). These results indicate that ZBTB20 has a promotional effect on HDPSC differentiation into odontoblasts.

ZBTB20 promotes odontogenic differentiation of HDPSCS via activation of the NF- $\kappa B$ pathway

As a transcriptional factor, ZBTB20 may regulate odontogenic differentiation via

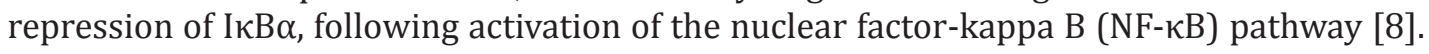


A
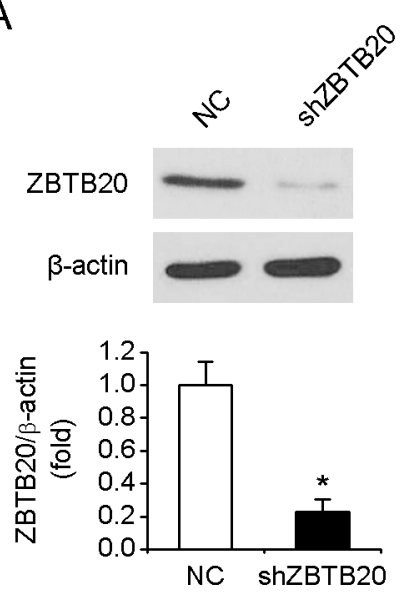

B

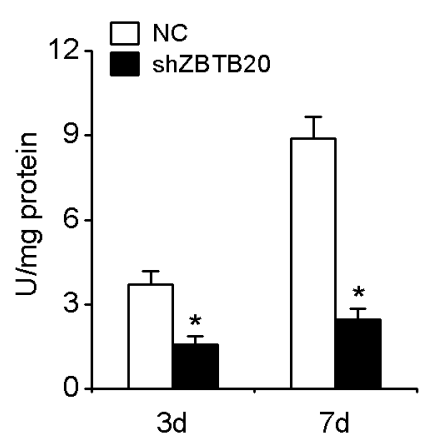

D
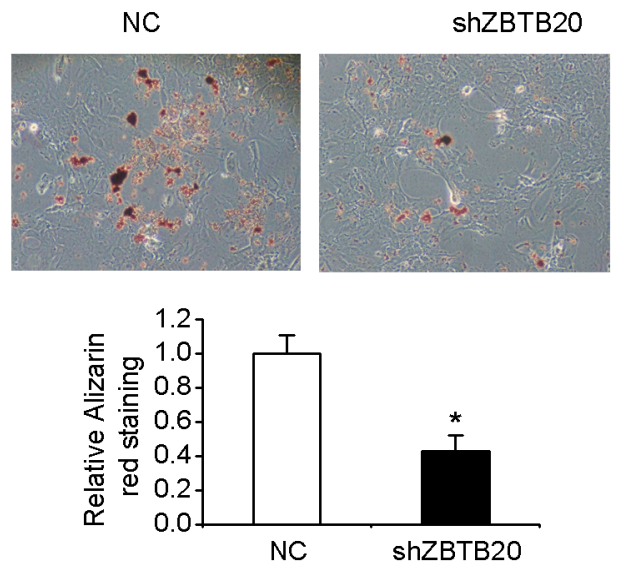

C

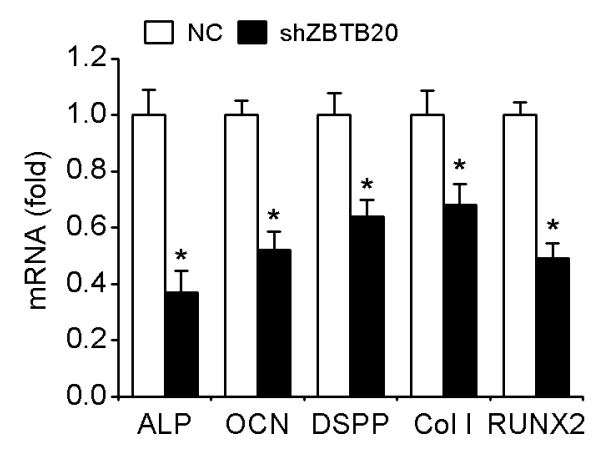

Fig. 2. Inhibition of ZBTB20 impaired odontogenic differentiation of HDPSCs. (A) Western blot analysis of ZBTB20 expression in HDPSCs transfected with lentiviral constructs containing ZBTB20 shRNA or negative control. Protein levels were normalized as a ratio to $\beta$-actin after densitometric quantification and presented as fold change relative to the control group. The above cells were treated with OM for 14 days. Odontogenic differentiation was assessed by measuring ALP activity at 3 and 7 days (B), mRNA expression levels of ALP, OCN, DSPP, Col I and RUNX2 at 7 days (C), and Alizarin red staining at 14 days (D). Values are presented as mean \pm SD for three independent experiments. ${ }^{*} P<0.05$ vs. NC group.

To investigate this, expression of IкB $\alpha$ and p65 were detected by western blot following incubation of HDPSCs silencing or overexpressing ZBTB20 in OM for $48 \mathrm{~h}$. As shown in Fig. $4 \mathrm{~A}$ and $\mathrm{B}, \mathrm{ZBTB} 20$ inhibition significantly increased $\mathrm{I} \kappa \mathrm{B} \alpha$ expression compared to the control cells, while ZBTB20 overexpression decreased IкB $\alpha$ expression and increased nuclear $\mathrm{p} 65$. To ascertain whether p65 nuclear translocation occurred following exposure to ZBTB20, an immunofluorescence assay was performed to confirm nuclear localization. The results showed that ectopic ZBTB20 expression caused the translocation of NF- $\kappa$ B p65 from the cytoplasm to cell nuclei. These results suggest that ZBTB20 activates the NF- $\mathrm{kB}$ pathway in HDPCs during odontogenic differentiation.

Furthermore, we investigated whether activation of the NF- $\kappa \mathrm{B}$ pathway is critical for the induction of odontogenic differentiation by ZBTB20 in HDPSCs. BMS-345541 (a highly selective inhibitor of NF- $\kappa$ B) was used to block the phosphorylation of IкB $\alpha$ and p65 [20-22], following which ALP activity and expression levels of several gene markers of differentiation were assessed. As expected, BMS-345541 significantly inhibited the increase of ALP activity and the mRNA expression of ALP, OCN, DSPP, Col I and RUNX2 induced by ZBTB20 (Fig. $5 \mathrm{~A}-\mathrm{C}$ ), indicating the involvement of the NF- $\kappa \mathrm{B}$ signaling pathway. 
A
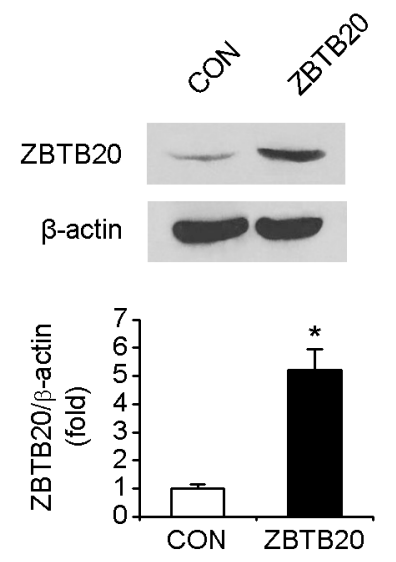

B

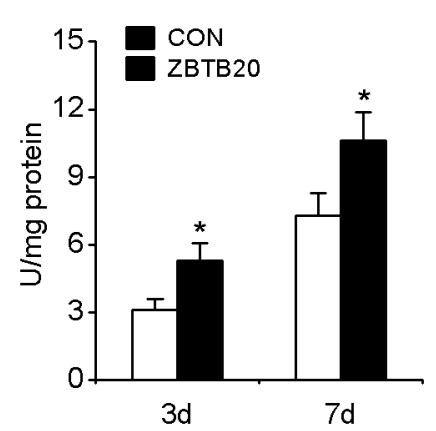

$\mathrm{D}$
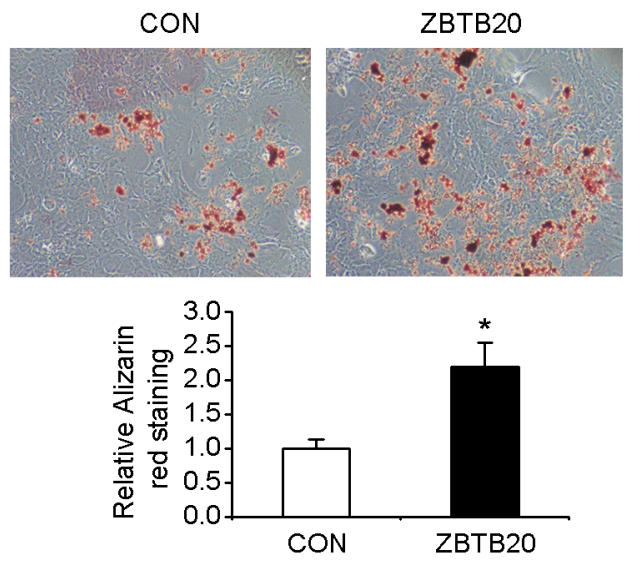

C

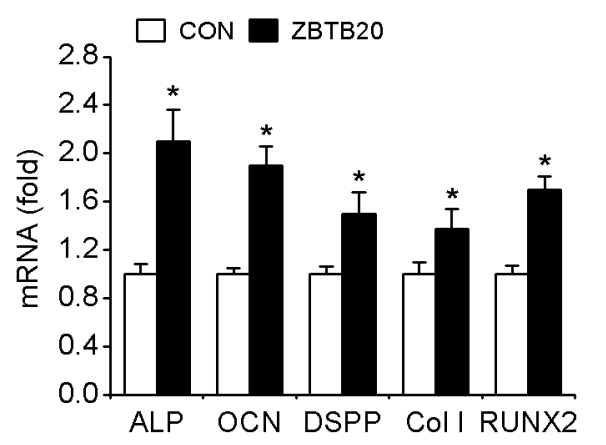

Fig. 3. Overexpression of ZBTB20 promotes odontogenic differentiation of HDPSCs. (A) ZBTB20-expressing lentiviruses were transduced into HDPSCs, and ZBTB20 expression was confirmed by western blot. Protein levels were normalized as a ratio to $\beta$-actin after densitometric quantification and presented as fold change relative to the control group. These cells were then treated with OM for 14 days. Odontogenic differentiation was assessed by measuring ALP activity (B), mRNA expression levels of ALP, OCN, DSPP, Col I and RUNX2 (C), and Alizarin red staining (D). Values are presented as mean \pm SD for three independent experiments. ${ }^{*} P$ $<0.05$ vs. control group.

\section{Discussion}

The potential of HDPSCs for use in induction of reparative dentin has been recognized because of their odontogenic differentiation potential [4]. However, the molecular mechanisms underlying their odontogenic differentiation remain unclear. In this study, we show that the transcription factor ZBTB20 is significantly upregulated in a time-dependent manner during odontogenic differentiation. This indicates that ZBTB20 may act as a positive regulator of odontogenic differentiation. Silencing of ZBTB20 reduced ALP activity, mineralization and the expression of differentiation marker genes. In contrast, overexpression of ZBTB20 enhanced OM-induced odontogenic differentiation. Furthermore, we show that the NF- $\mathrm{BB}$ pathway is critical for ZBTB20-mediated odontoblastic differentiation of HDPSCs. This is the first study to characterize ZBTB20 regulation in HDPSCs and to identify ZBTB20 as one of the critical factors in odontogenic differentiation.

ALP provides a source of inorganic phosphate for hydroxyapatite formation, which is required for the proper mineralization of bone [23]. ALP knockout mice exhibit defects in bone mineralization [24], demonstrating that ALP is an important early-stage marker of osteoblast/odontoblast differentiation. OCN is one of the major noncollagenous proteins 
Fig. 4. Effects of ZBTB20 inhibition and overexpression on the $\mathrm{NF}-\kappa \mathrm{B}$ pathway during HDPSC differentiation. HDPSCs with ZBTB20 inhibition or overexpression were incubated in OM for $60 \mathrm{~min}$. (A) Expression of I $\mathrm{I} B \alpha$ was determined by western blot. Protein levels were normalized as a ratio to $\beta$-actin after densitometric quantification and presented as fold change relative to the control group. (B) Expression of nuclear NF- $\mathrm{B}$ p 65. Lamin-B was used as an internal control. (C) Nuclear localization of NF- $\kappa$ B p65 was detected by immunofluorescent staining.

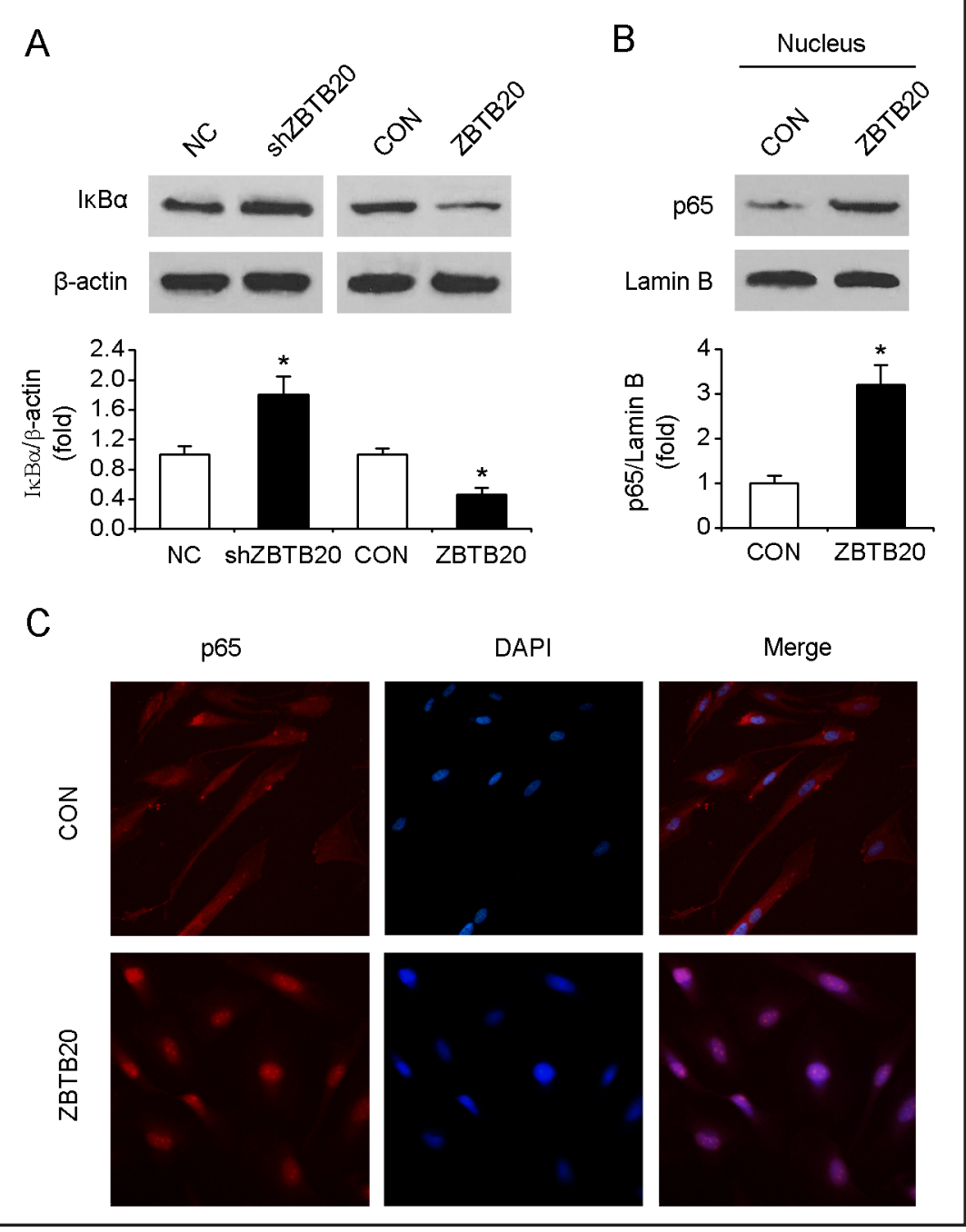

B

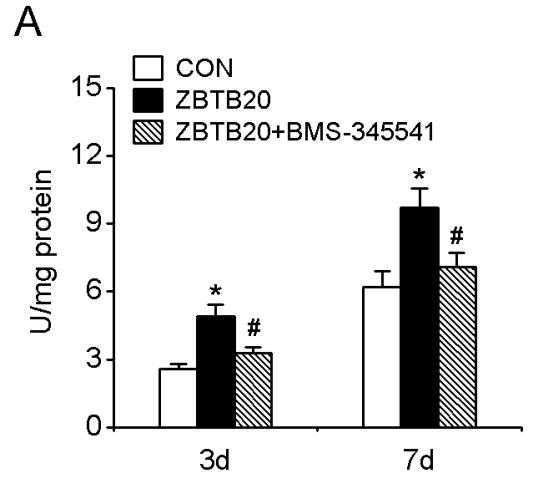

Fig. 5. Inhibition of ZBTB20-induced odontogenic differentiation by BMS-345541. Cells were cultured in OM with or without BMS-345541 (1 $\mu \mathrm{mol} / \mathrm{L})$. (A) ALP activity was measured at 3 and 7 days. (B) The mRNA levels of ALP, OCN, DSPP, Col I and RUNX2 were detected by qRT-PCR at 7 days. ${ }^{*} P<0.05$ vs. control group, ${ }^{\#} P<0.05$ vs. ZBTB20 group.

specific to mineralized connective tissues of vertebrates [25], while DSPP and Col I have been reported to be highly expressed in odontoblasts and essential for dentinogenesis $[26,27]$. OCN, DSPP and Col I mainly appear during the late stages of odontoblastic differentiation. 
Additionally, studies have suggested that the transcription factor RUNX2 is involved in osteoblast and odontoblast differentiation at the early and later stages [28, 29]. To assess the role of ZBTB20 in odontoblastic differentiation of HDPSCs, we examined the effects of ZBTB20 inhibition and overexpression on the expression of key differentiation markers. Our results in dicate that ZBTB20 inhibition significantly downregulates ALP activity, mineralized nodule formation, and the expression of early and late odontoblastic differentiation markers, including ALP, OCN, DSPP, Col I and RUNX2. Furthermore, ZBTB20 overexpression increases $\mathrm{OM}$-induced odontoblastic differentiation. Our results are consistent with the finding in a recent study that ZBTB20 is high expressed in hypertrophic chondrocytes and that deletion of ZBTB20 in developing cartilage results in delayed endochondral ossification and postnatal growth retardation [30].

The NF- $\kappa \mathrm{B}$ pathway has been reported to be extensively involved in differentiation of osteoblasts and odontoblasts [31,32]. For example, Hess et al. showed that enhanced NF- $\kappa B$ activity in human mesenchymal stem cells increases osteogenic differentiation [33]. Li et al. reported that NF- $\kappa$ B pathway-activated human stem cells from apical papilla exhibit higher proliferative activity, together with enhanced odonto/osteogenic ability [34]. IкB $\alpha$ protein is the major inhibitor of the NF- $\kappa \mathrm{B}$ pathway. In its inactive state, NF- $\kappa \mathrm{B}$ protein binds to $\mathrm{I} \kappa \mathrm{B}$ family proteins (most importantly the I $\mathrm{B} \alpha$ protein) in the cytoplasm. Upon activation of the NF- $\kappa B$ pathway, I $\kappa \mathrm{B} \alpha$ is phosphorylated, ubiquitinated and degraded, which releases $\mathrm{NF}-\kappa \mathrm{B}$ to translocate to the nucleus and activate transcription of NF- $\kappa \mathrm{B}$-regulated genes $[35,36]$. A recent study showed that ZBTB20 specifically binds to the IкB $\alpha$ gene promoter, repressing І $\kappa \mathrm{B} \alpha$ gene transcription, and inhibits Iк $\mathrm{B} \alpha$ protein expression, thus promoting NF- $\kappa B$ activation [8]. In light of this, we next investigated whether ZBTB20 influenced the expression of IKB $\alpha$ and p65. We found that ZBTB20 overexpression significantly reduced IкB $\alpha$ expression and increased nuclear p65 during odontogenic differentiation, suggesting that ZBTB20 influences HDPSC differentiation through the NF- $\kappa B$ pathway. This finding was further supported by the fact that BMS-345541 blocked ZBTB20-induced ALP activity, mineralized nodule formation, and activation of ALP, OCN, DSPP, Col I and RUNX2. These data indicate that the NF- $\kappa$ B pathway is involved in the ZBTB20-mediated regulation of odontogenic differentiation of HDPSCs.

In summary, our results demonstrate that ZBTB20 promotes the odonto/osteogenic differentiation of HDPSCs, at least in part, through activation of the NF- $\kappa B$ pathway. This suggests that ZBTB20 may be a novel and important modulator of HDPSC differentiation, and could be useful in dental tissue regeneration and tissue engineering.

\section{Acknowledgments}

This study was funded by The National Natural Science Foundation of China (No. 81300865).

\section{Disclosure Statement}

None declared.

\section{References}

1 Gronthos S, Mankani M, Brahim J, Robey PG, Shi S: Postnatal human dental pulp stem cells (dpscs) in vitro and in vivo. Proc Natl Acad Sci U S A 2000;97:13625-13630.

2 Huang GT, Yamaza T, Shea LD, Djouad F, Kuhn NZ, Tuan RS, Shi S: Stem/progenitor cell-mediated de novo regeneration of dental pulp with newly deposited continuous layer of dentin in an in vivo model. Tissue Eng Part A 2010;16:605-615. 


\section{Cellular Physiology Cell Physiol Biochem 2015;36:1725-1734 \begin{tabular}{l|l} 
and Biochemistry $\begin{array}{l}\text { DOI 10.1159/000430145 } \\
\text { Published online: July 13, } 2015\end{array}$ & $\begin{array}{l}\text { C 2015 S. Karger AG, Basel } \\
\text { www.karger.com/cpb }\end{array}$ \\
\hline
\end{tabular} \\ Gu et al.: ZBTB20 Regulates Odontogenic Differentiation of hDPSCs}

3 Miura M, Gronthos S, Zhao M, Lu B, Fisher LW, Robey PG, Shi S: Shed: Stem cells from human exfoliated deciduous teeth. Proc Natl Acad Sci U S A 2003;100:5807-5812.

4 Iohara K, Zheng L, Ito M, Tomokiyo A, Matsushita K, Nakashima M: Side population cells isolated from porcine dental pulp tissue with self-renewal and multipotency for dentinogenesis, chondrogenesis, adipogenesis, and neurogenesis. Stem Cells 2006;24:2493-2503.

5 Zhang W, Walboomers XF, Van Kuppevelt TH, Daamen WF, Van Damme PA, Bian Z, Jansen JA: In vivo evaluation of human dental pulp stem cells differentiated towards multiple lineages. J Tissue Eng Regen Med 2008;2:117-125.

6 Liu H, Gronthos S, Shi S: Dental pulp stem cells. Methods Enzymol 2006;419:99-113.

7 Rosenthal EH, Tonchev AB, Stoykova A, Chowdhury K: Regulation of archicortical arealization by the transcription factor zbtb20. Hippocampus 2012;22:2144-2156.

8 Liu X, Zhang P, Bao Y, Han Y, Wang Y, Zhang Q, Zhan Z, Meng J, Li Y, Li N, Zhang WJ, Cao X: Zinc finger protein zbtb20 promotes toll-like receptor-triggered innate immune responses by repressing ikappabalpha gene transcription. Proc Natl Acad Sci U S A 2013;110:11097-11102.

9 Sutherland AP, Zhang H, Zhang Y, Michaud M, Xie Z, Patti ME, Grusby MJ, Zhang WJ: Zinc finger protein zbtb20 is essential for postnatal survival and glucose homeostasis. Mol Cell Biol 2009;29:2804-2815.

10 Zhang Y, Xie Z, Zhou L, Li L, Zhang H, Zhou G, Ma X, Herrera PL, Liu Z, Grusby MJ, Zhang WJ: The zinc finger protein zbtb20 regulates transcription of fructose-1,6-bisphosphatase 1 and beta cell function in mice. Gastroenterology 2012;142:1571-1580 e1576.

11 Ren AJ, Wang K, Zhang H, Liu A, Ma X, Liang Q, Cao D, Wood JN, He DZ, Ding YQ, Yuan WJ, Xie Z, Zhang WJ: Zbtb20 regulates nociception and pain sensation by modulating trp channel expression in nociceptive sensory neurons. Nat Commun 2014;5:4984.

12 Zhang W, Mi J, Li N, Sui L, Wan T, Zhang J, Chen T, Cao X: Identification and characterization of dpzf, a novel human btb/poz zinc finger protein sharing homology to bcl-6. Biochem Biophys Res Commun 2001;282:1067-1073.

13 Felthaus O, Gosau M, Klein S, Prantl L, Reichert TE, Schmalz G, Morsczeck C: Dexamethasone-related osteogenic differentiation of dental follicle cells depends on zbtb16 but not runx2. Cell Tissue Res 2014;357:695-705.

14 Ikeda R, Yoshida K, Tsukahara S, Sakamoto Y, Tanaka H, Furukawa K, Inoue I: The promyelotic leukemia zinc finger promotes osteoblastic differentiation of human mesenchymal stem cells as an upstream regulator of cbfa1. J Biol Chem 2005;280:8523-8530.

15 Inoue I, Ikeda R, Tsukahara S: Current topics in pharmacological research on bone metabolism: Promyelotic leukemia zinc finger (plzf) and tumor necrosis factor-alpha-stimulated gene 6 (tsg-6) identified by gene expression analysis play roles in the pathogenesis of ossification of the posterior longitudinal ligament. J Pharmacol Sci 2006;100:205-210.

16 Felthaus 0, Gosau M, Morsczeck C: Zbtb16 induces osteogenic differentiation marker genes in dental follicle cells independent from runx2. J Periodontol 2014;85:e144-151.

17 Choi YA, Seol MY, Shin HI, Park EK: Bobby sox homology regulates odontoblast differentiation of human dental pulp stem cells/progenitors. Cell Commun Signal 2014;12:35.

18 Qu B, Liu O, Fang X, Zhang H, Wang Y, Quan H, Zhang J, Zhou J, Zuo J, Tang J, Tang Z: Distal-less homeobox 2 promotes the osteogenic differentiation potential of stem cells from apical papilla. Cell Tissue Res 2014;357:133-143.

19 Go HS, Seo JE, Kim KC, Han SM, Kim P, Kang YS, Han SH, Shin CY, Ko KH: Valproic acid inhibits neural progenitor cell death by activation of nf-kappab signaling pathway and up-regulation of bcl-xl. J Biomed Sci 2011;18:48.

20 Yang J, Amiri KI, Burke JR, Schmid JA, Richmond A: Bms-345541 targets inhibitor of kappab kinase and induces apoptosis in melanoma: Involvement of nuclear factor kappab and mitochondria pathways. Clin Cancer Res 2006;12:950-960.

21 Burke JR, Pattoli MA, Gregor KR, Brassil PJ, MacMaster JF, McIntyre KW, Yang X, Iotzova VS, Clarke W, Strnad J, Qiu Y, Zusi FC: Bms-345541 is a highly selective inhibitor of i kappa b kinase that binds at an allosteric site of the enzyme and blocks nf-kappa b-dependent transcription in mice. J Biol Chem 2003;278:1450-1456. 


\section{Cellular Physiology Cell Physiol Biochem 2015;36:1725-1734 \begin{tabular}{ll|l} 
and Biochemistry $\begin{array}{l}\text { DOI: 10.1159/000430145 } \\
\text { Published online: July 13, 2015 }\end{array}$ & $\begin{array}{l}\text { O) 2015 S. Karger AG, Basel } \\
\text { www.karger.com/cpb }\end{array}$ \\
\cline { 2 - 3 } & Gu
\end{tabular} \\ Gu et al.: ZBTB20 Regulates Odontogenic Differentiation of hDPSCs}

22 Wang Y, Zheng Y, Wang Z, Li J, Zhang G, Yu J: 10(-7) m 17beta-oestradiol enhances odonto/osteogenic potency of human dental pulp stem cells by activation of the nf-kappab pathway. Cell Prolif 2013;46:677684.

23 Harrison G, Shapiro IM, Golub EE: The phosphatidylinositol-glycolipid anchor on alkaline phosphatase facilitates mineralization initiation in vitro. J Bone Miner Res 1995;10:568-573.

24 Wennberg C, Hessle L, Lundberg P, Mauro S, Narisawa S, Lerner UH, Millan JL: Functional characterization of osteoblasts and osteoclasts from alkaline phosphatase knockout mice. J Bone Miner Res 2000;15:18791888.

25 Neugebauer BM, Moore MA, Broess M, Gerstenfeld LC, Hauschka PV: Characterization of structural sequences in the chicken osteocalcin gene: Expression of osteocalcin by maturing osteoblasts and by hypertrophic chondrocytes in vitro. J Bone Miner Res 1995;10:157-163.

26 Yamakoshi Y: Dentinogenesis and dentin sialophosphoprotein (dspp). J Oral Biosci 2009;51:134.

27 Chen S, Gluhak-Heinrich J, Wang YH, Wu YM, Chuang HH, Chen L, Yuan GH, Dong J, Gay I, MacDougall M: Runx2, osX, and dspp in tooth development. J Dent Res 2009;88:904-909.

28 Nakashima K, Zhou X, Kunkel G, Zhang Z, Deng JM, Behringer RR, de Crombrugghe B: The novel zinc fingercontaining transcription factor osterix is required for osteoblast differentiation and bone formation. Cell 2002;108:17-29.

29 Celil AB, Campbell PG: Bmp-2 and insulin-like growth factor-i mediate osterix (osx) expression in human mesenchymal stem cells via the mapk and protein kinase d signaling pathways. J Biol Chem 2005;280:31353-31359.

30 Zhou G, Jiang X, Zhang H, Lu Y, Liu A, Ma X, Yang G, Yang R, Shen H, Zheng J, Hu Y, Yang X, Zhang WJ, Xie Z: Zbtb20 regulates the terminal differentiation of hypertrophic chondrocytes via repression of sox9. Development 2015;142:385-393.

31 Chang J, Zhang C, Tani-Ishii N, Shi S, Wang CY: Nf-kappab activation in human dental pulp stem cells by tnf and lps. J Dent Res 2005;84:994-998.

32 Lee YM, Shin SY, Jue SS, Kwon IK, Cho EH, Cho ES, Park SH, Kim EC: The role of pin1 on odontogenic and adipogenic differentiation in human dental pulp stem cells. Stem Cells Dev 2014;23:618-630.

33 Hess K, Ushmorov A, Fiedler J, Brenner RE, Wirth T: Tnfalpha promotes osteogenic differentiation of human mesenchymal stem cells by triggering the nf-kappab signaling pathway. Bone 2009;45:367-376.

34 Li J, Yan M, Wang Z, Jing S, Li Y, Liu G, Yu J, Fan Z: Effects of canonical nf-kappab signaling pathway on the proliferation and odonto/osteogenic differentiation of human stem cells from apical papilla. Biomed Res Int 2014;2014:319651.

35 Karin M, Ben-Neriah Y: Phosphorylation meets ubiquitination: The control of nf-[kappa]b activity. Annu Rev Immunol 2000;18:621-663.

36 Bonizzi G, Karin M: The two nf-kappab activation pathways and their role in innate and adaptive immunity. Trends Immunol 2004;25:280-288. 
In the article by the authors: Gu et al., entitled "Human Dental Pulp Stem Cells via the NF- $\kappa B$ Pathway" [Cell Physiol Biochem 2015;36:1725-1734 (DOI: 10.1159/000430145)], there is an error in title.

The correct title is reproduced here:

"ZBTB20 regulates odontogenic differentiation of human dental pulp stem cells via the NF$\kappa \mathrm{B}$ pathway"

The authors sincerely apologize for this error. 